\title{
Strain rate dependence of Ti64: characterisation of mechanical properties up to failure using novel optical techniques
}

\author{
M. Arthington ${ }^{1}$, C. Siviour ${ }^{1}$, N. Petrinic ${ }^{1}$ and B. Elliott ${ }^{1}$ \\ ${ }^{1}$ University of Oxford, Dept. Engineering Science, Parks Road, Oxford
}

\begin{abstract}
Two methods for the improved characterisation of anisotropic materials are presented. One method utilises three optical videos of cylindrical specimens undergoing uniaxial loading to reconstruct elliptical cross-sectional contours. This information can be used to improve knowledge of anisotropic plastic deformation behaviour. A second method uses digital images of specimens to perform the task of a video extensometer. Through the high resolution imaging of specimens, the undulations due to machining can be seen in edges detected to sub-pixel accuracy. These edges have unique variations along their length that may be tracked to provide high density strain data without occlusions due to the marking required by other methods. The cross-section reconstruction has been applied to rolled Ti64 at strain rates of $\sim 10^{-3} \mathrm{~s}^{-1}, \sim 1 \mathrm{~s}^{-1}$ and $\sim 10^{3} \mathrm{~s}^{-1}$ in compression and tension experiments. Three views have been obtained using both multiple camera rigs and mirrors. The strain measurement through edge tracking has been applied to specimens loaded at $\sim 10^{-3} \mathrm{~s}^{-1}$ using high resolution imaging.
\end{abstract}

\section{INTRODUCTION}

Ti6Al4V is a widely used alloy in aerospace and bioengineering applications where its relatively high cost is justified. Desirable properties such as resistance to corrosion, high ductility, high strength to weight ratio and energy absorption capabilities under impact have led to its use in products such as replacement hip joints and jet engine blades. Due to its high raw material cost relative to other metals such as steel and Aluminium, and often the weight constraint of the application, engineers are encouraged to reduce the amount of Ti64 used. In order to reduce costly experiments, the products are often modelled using the finite element method to predict their likely in-service behaviour.

The material models that are employed in numerical modelling are only as good as the principles on which they operate and the parameters that are provided through material characterisation. Models for Ti64 are most often based on isotropic behaviour, however these cannot capture the orthotropic behaviour of rolled plates to a sufficient degree for highly accurate simulations.

This paper outlines two experimental techniques using digital imaging that have been developed to capture the behaviour of cylindrical specimens at a wide range of strain rates and uniaxial loading directions.

\subsection{The elliptical deformation of rolled plate specimens}

When an initially isotropic material has been plastically deformed during its manufacture, the material will behave anisotropically during further loading in the plastic regime. This anisotropy presents itself as an increasingly elliptical cross-section with increasing plastic strain in uniaxially loaded, initially cylindrical, specimens. This deformation behaviour may be quantified to provide an extra source of information to determine material parameters for models. Optical techniques for 
measuring two dimensional geometry from a single view of specimens are commonplace, e.g. [1], however a means of constructing the $3 \mathrm{D}$ specimen shape from $2 \mathrm{D}$ images is required for anisotropic deformation of specimens. The observable differences in radii in different views can be seen in Figure 1(a). Reconstruction techniques for applications similar to this, [2], have been developed where the specimen is rotated to give 3D shapes from occluded regions, however this doesn't make use of the knowledge of the deforming shape contour (an ellipse), and could not be implemented at high strain rates.

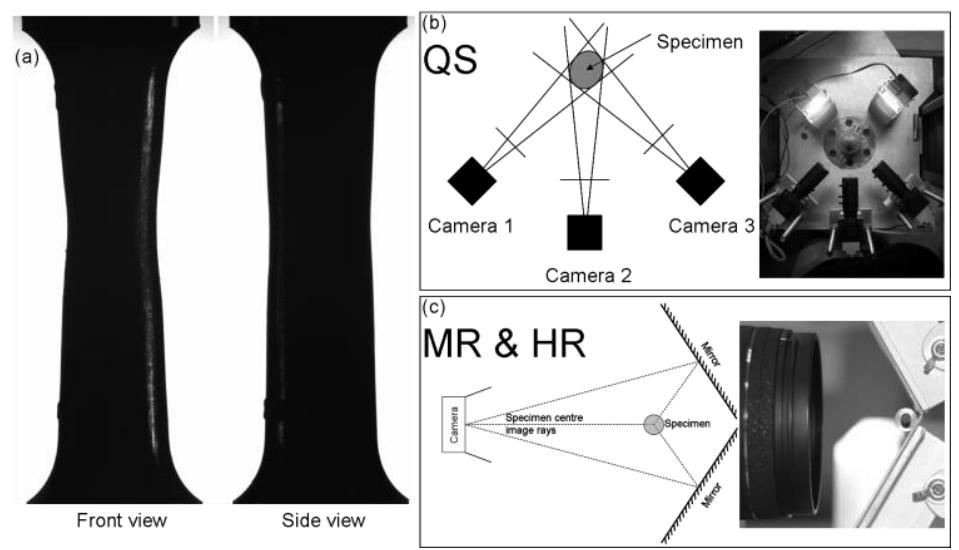

Figure 1: (a) two views of a specimen deformed at a quasi-static (QS) rate in the last frame before fracture. The different diameters at the neck demonstrate the anisotropy. (b) and (c) show the multi-camera set-up and the single camera with mirrors set-up used in QS and medium and high strain rate experiments (MR and HR). Mirrored views have depth of field disparities between views, but do not require simultaneous triggering like multiple camera views do.

In order to measure the elliptical cross section of a specimen at many strain rates, an optical technique has been developed whereby silhouette images of a specimen are captured throughout an experiment from three views, as shown in Figure 1(b) and 1(c). The positions of the cameras and the positions of the edges in those views define bounding tangential lines to the cross-section contours, as shown in Figure 1(b), at all distinguishable heights along the specimen axis.

If the cameras are sufficiently far from the specimen, the six lines may be considered to lie on cross-sectional planes. In each of these planes, an ellipse can be fitted to the lines which minimises the sum of the squared conic equation, formulated in terms of the bounding line equations. This results in a non-linear least squares fitting that can be extended to include many tangent rays, from as many views as can be obtained, to produce the most accurate results. And conversely, five lines is the minimum number required to define an arbitrary ellipse.

In order to determine these tangential lines accurately from experiments, the cameras need to be intrinsically and extrinsically calibrated using techniques outlined in, for example, [3]. The edges of the specimen also need to be determined as accurately as possible and a sub-pixel edge detection algorithm was written that detected the edges at the locations of the maximum gradient changes in each row of an image. The results of this work applied to experiments on Ti64 at strain rates of $10^{-3} \mathrm{~s}^{-1}$ and $10^{3} \mathrm{~s}^{-1}$ can be seen in Section 2.

\subsection{Video extensometry through machining-mark tracking}

Commercially available video extensometers provide a versatile, non-contacting, means of recording displacements in experiments. However, one of the drawbacks of video extensometers is that the surface being tracked has to have sufficient contrast between adjacent points to tell them apart. In some materials a surface texture is inherent, such as in some composites, unpolished stones etc. However in general, macroscopically viewed metallic specimens have surfaces that appear uniform and often have to be marked with a material such as paint to provide contrasting points for tracking. 


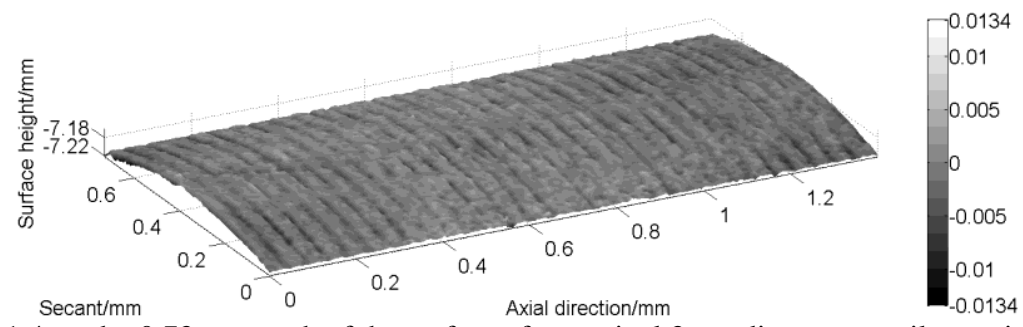

Figure 2: A $1.4 \mathrm{~mm}$ by $0.73 \mathrm{~mm}$ patch of the surface of a nominal $3 \mathrm{~mm}$ diameter tensile specimen produced using an Alicona InfiniteFocus Surface Profiler. The shading denotes height deviation from cylindrical, in $\mathrm{mm}$.

Marking the specimens of Ti64 under consideration here would obscure the specimen edges, thus preventing accurate reconstruction of the cross-section wherever paint was applied. For this reason a novel method of displacement measurement was required.

Machining marks, which can be seen in the surface profile of Figure 2, are visible after the edge has been detected to a high accuracy at all rows, as shown in Figure 3. The visibility of the machining marks presents an opportunity for optical tracking that doesn't require marking of surfaces.

An algorithm was developed to track local maxima [and minima, which is the same process as for maxima but taking the negative edge] in the detected edge profile, as shown in Figure 3. These maxima are defined to be points where the radius is greater than the two surrounding points. The positions of these maxima are tracked by searching for the closest maxima in a subsequent frame. As the surface geometry changes throughout the experiment, not all of the maxima are visible in all frames, resulting in the loss of tracking for some maxima.

Filtering, which can take several forms, has been applied to remove the 'bad' tracks. The filtering developed for this purpose included: removing all tracks that have a movement between two frames greater than a given maximum; tracking all maxima forward in time and then backwards, the tracks that are the same in both directions are visible throughout the experiment; and applying a directional constraint when it was known that in certain frames,

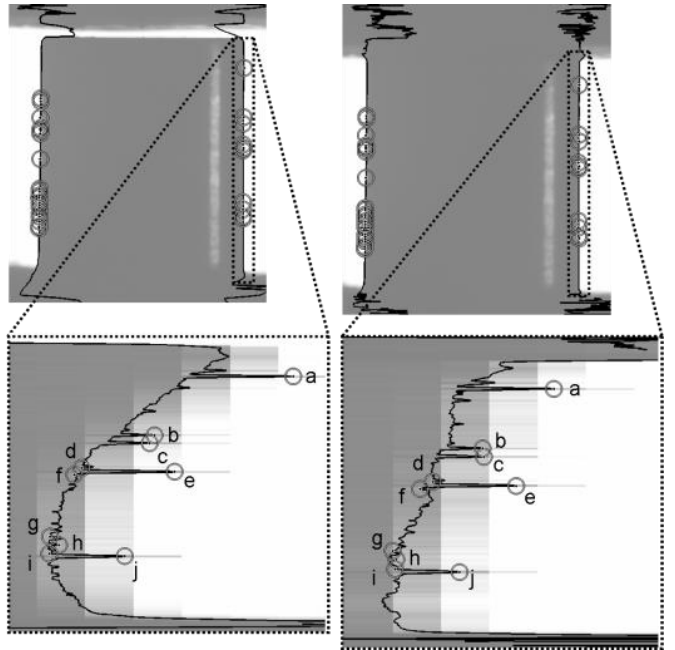

Figure 3: A 3.5mm long QS compression specimen before loading and at a time step where the nominal strain is $-2.7 \%$, the specimen has been faded from the original image for clarity. The magnified regions shows the sub-pixel-detected edge and tracked local maxima and minima of the machining marks. maxima may only move in a certain direction, for instance in the compression experiment of Figure 3, all the maxima have to move downwards during the entire experiment. Figure 3 shows the positions of maxima and minima tracks between the first frame and a frame 40 time steps later, where the nominal strain has reached $-2.7 \%$. It can be observed in Figure 3 that there are many more local maxima than those highlighted, but filtering has removed those maxima that could not be tracked throughout the experiment.

An example of the strain measurable, using the tracks of Figure 3, is shown in Figure 7. The strain is compared in Figure 7 to that calculated using a laser extensometer and paint applied up to the edges of the compression anvils immediately above and below the specimen.

\section{ELLIPTICAL CROSS-SECTION RECONSTRUCTION OF TI64 AT THREE STRAIN RATES}

Uniaxial tensile experiments on cylindrical dog-bone specimens and compression experiments on cylindrical specimens of Ti64 were performed at strain rates of $\sim 10^{-3} \mathrm{~s}^{-1}$ (quasi-static, QS), $\sim 1 \mathrm{~s}^{-1}$ 
(medium strain rate MR) and $\sim 10^{3} \mathrm{~s}^{-1}$ (high strain rate, HR). The QS specimens were loaded using a screw driven Hounsfield tensometer and imaged using three TELI Digital Cameras (CSB4000CL) with 4 megapixel 10-bit greyscale CMOS sensors, using Computar Megapixel lenses. The MR specimens were loaded using an in-house hydraulic machine and imaged using a Phantom high speed camera. The HR specimens were loading using Split Hopkinson Bars [3] and imaged using a Specialised Imaging SIM16 high speed camera. Both MR and HR experiments used two mirrors to provide the necessary three views, as shown in Figure 1(c).

Figure 5 shows the reconstruction of a QS tensile specimen where a direction perpendicular to the axis has a lower direct yield stress than the other two directions. Figure 6 shows a HR specimen reconstruction of the same sample type, where increased localisation of the strain can be observed.
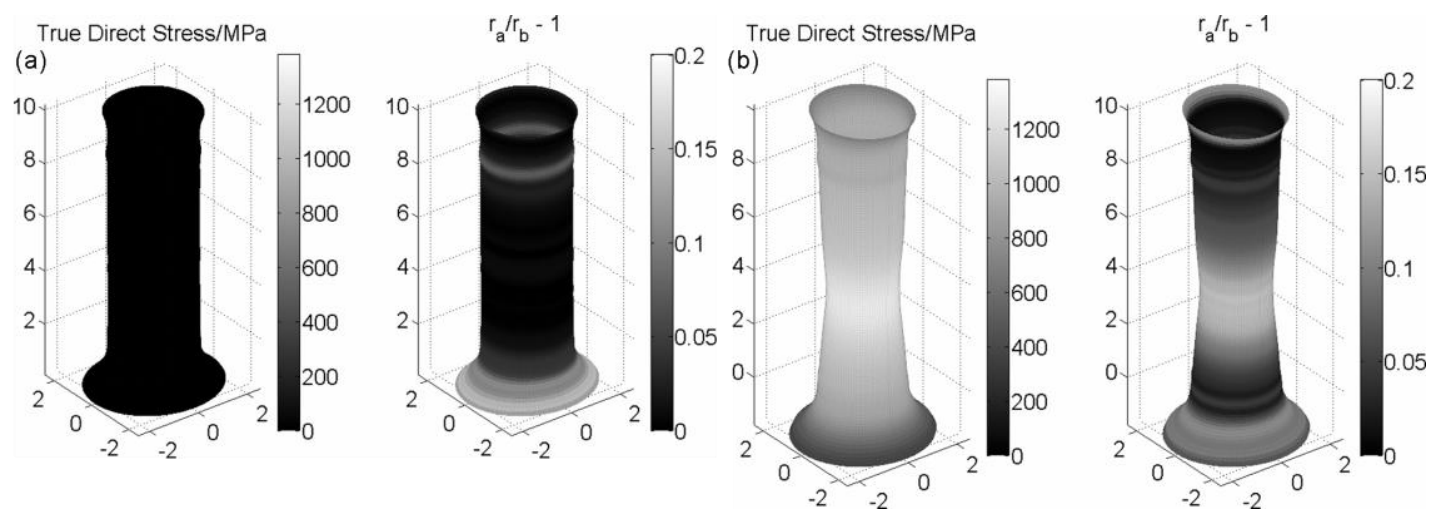

Figure 5: A QS tensile specimen before loading and at the last time recorded before fracture. Paint was used for this specimen to simultaneously measure strain using a laser extensometer, and the effect of this paint on the resultant cross-section measurement is highlighted by the elliptical region in (a) near the top. Other elliptical regions near the shoulders are due to noise in the edge data.

Figure 6 shows a specimen of the same design as in Figure 5, but loaded at HR.
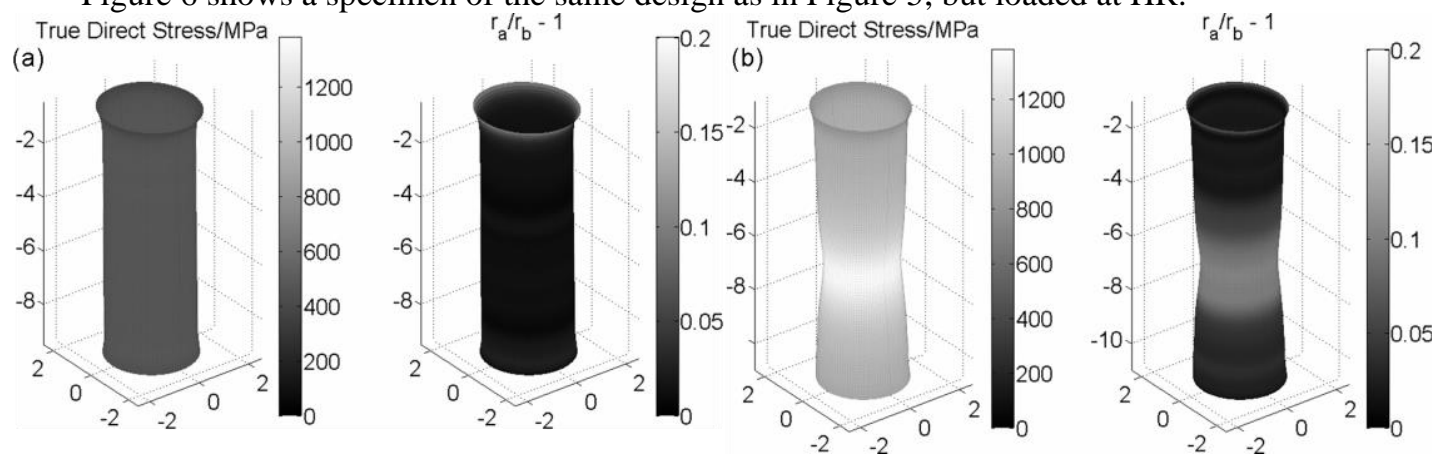

Figure 6: A HR tensile specimen near the start of the elastic region, (a), and at the last frame recorded before fracture, (b).

\section{EDGE TRACKING OF HIGH RESOLUTION MACROSCOPIC IMAGES FOR STRAIN MEASUREMENT}

Through the high resolution, sub-pixel edge detection mentioned in Section 1.2, it is possible to view the undulations of machining marks created during the turning of the specimens from their parent plates.

Using the images from the experiments performed in Section 2, the technique of tracking the undulations due to machining marks has been applied in QS experiments. In MR and HR experiments the resolutions of the cameras were too low at the magnification used to provide sufficiently detailed images, and hence edge information, for tracking. It is hoped that with improving high speed camera technology, higher resolution images will be available in the future. 
Figure 7 shows the strain results with time of edge tracking in a compression specimen of Ti64. The compliance of the load chain has resulted in the laser extensometer strain having a non-linear elastic region at first, and subsequently lower strain throughout. The quantisation noise in the machining mark strain measurement arises due to the discrete pixel tracking, and it is hoped that a sub-pixel search will be implemented to improve upon this, although simple low-pass filtering could be applied.
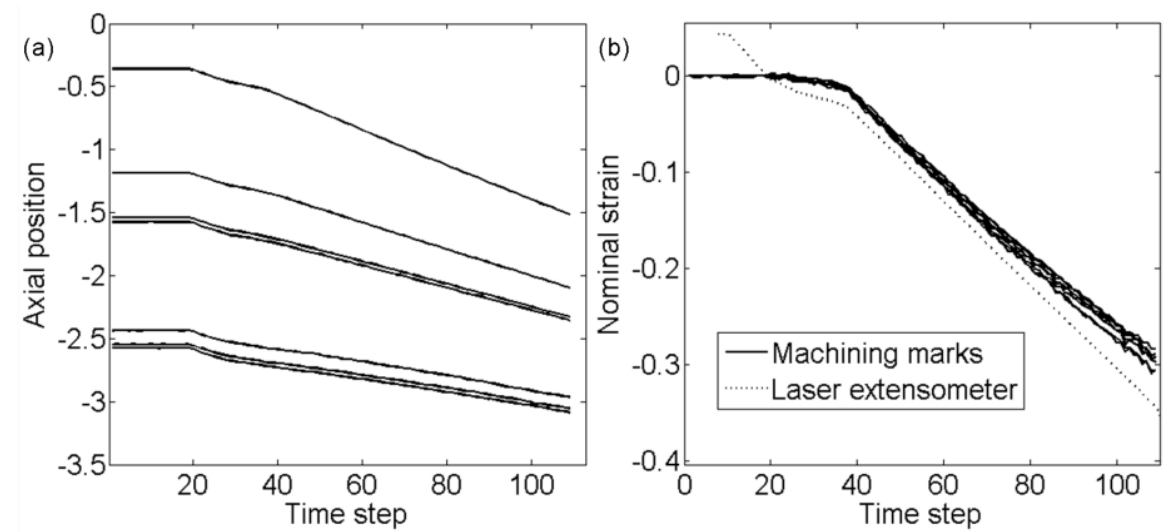

Figure 7: (a) shows the axial positions of the seven best tracked machining mark maxima and minima highlighted in Figure 3. (b) shows the strain from the laser extensometer and the six nominal strains taken between the top most track and the other tracks from (a). The anvils and the specimen came into contact at time step 19, where the plots intersect.

\section{DISCUSSION}

\subsection{Cross-sectional reconstruction}

The cross sectional reconstruction method provides non-contacting, in-situ measurement of anisotropy in both tension and compression. This is especially valuable in measuring plate material properties where a through thickness specimen cannot be obtained due to the thinness of the manufactured plate.

One drawback of the cross-sectional reconstruction is the degree to which the ellipticity is sensitive to noise on the measurements of edge positions. As stated earlier, the cameras must be calibrated accurately to rectify distortion. The images should be as sharp as possible with no over/under-exposure and they should have as little noise as possible which can interfere with the edge detection. In a recently submitted paper [4] the reconstruction technique is described in detail and evaluation of the method has been performed stochastically and through the measurement of elliptical prisms. It is shown that there are optimum view orientations that minimise the effects of noise by improving the conditioning of the data for the least squares fitting.

\subsection{Tracking for strain measurement}

As there are at least six specimen edge positions measured during an experiment, there are six sites where machining mark tracking may take place. This creates an abundance of precise position information in the gauge section that has not been available before without the use of high resolution extensometry and fine surface texture.

Whilst edge tracking may not be possible at all strain rates at present, the task of a video extensometer may still be performed with the sacrifice of obscuring the edges by painting. This tradeoff allows nearly continuous measurement of cross-section, whilst providing low density position tracking for strain measurement. The combination of paint and reconstruction has been used in MR and HR experiments to produce strain information which is better than the simple analysis of the support movements, but with a reconstruction of the cross-section that is not continuous.

The machining marks observed here, as shown in Figure 2, have been measured to have a root mean squared radius of $0.13 \%$ of the specimen radius. These small deviations are not expected to have 
a significant effect on the specimen's macroscopic deformation behaviour, but may have a minor effect on the localisation site of shear bands. The advantages of having them on the specimen outweigh any gains were the specimen to be polished.

\subsection{The combination of the techniques}

The combination of these measurement techniques creates 3 dimensional surface tracking of elliptical specimens. Whilst the current apparatus limits the extensometry to QS experiments, the crosssectional shape obtained at all rates provides useful data for inverse parameter identification in anisotropic models. Alternatively this data may prove useful in validation experiments where elliptical radial displacements have been predicted.

\section{CONCLUSIONS}

Two techniques for the improved characterisation of anisotropic materials at strain rates ranging from quasi-static to $10^{3} \mathrm{~s}^{-1}$ have been presented. One technique uses three silhouette views of a specimen throughout an experiment to obtain in-situ measurements of an elliptical cross-section with a high density of data in the axial direction. Another technique involves the use of machining marks from the manufacture of specimens, detected using a sub-pixel edge detection, to track positions on the surface of the specimen for high density strain information.

These techniques have been demonstrated using anisotropic specimens of Ti64, but would be equally valid for any homogeneous anisotropic material. At present the combination of the techniques can only be applied in quasi-static experiments where the resolution of the images is sufficient to capture the silhouette of the surface details as well as the full view of the specimen for reconstruction. However the methodology will be applicable to high strain rate experiments with improved technology and higher magnifications may be used to obtain high density machining mark tracking in high speed images.

The combination of both of the techniques provides full surface measurements of anisotropic, homogeneous, initially cylindrical specimens throughout an experiment. The machining mark tracking provides strain data that does not have the problems inherent in marking specimens with paint, such as surface occlusion, melting or non-adherence. Either the combination of both or the use of just the cross-section reconstruction may be used as validation of finite element models. Alternatively, through inverse identification techniques, anisotropic material parameters maybe obtained with a higher certainty of their values than before.

\section{Acknowledgments}

This work has been funded by the Engineering and Physical Sciences Research Council (EPSRC) and RollsRoyce plc. The Specialised Imaging SIM16 high speed camera used in this research was provided by the EPSRC Instrument Loan Pool. We are particularly grateful to A Walker for his advice and support during this loan. Many thanks also to colleagues at the University of Oxford, especially P Harding, G Hillsdon and D Mulvihill.

\section{References}

[1] C G'Sell. Video-controlled tensile testing of polymers and metals beyond the necking point. Journalof materials science, 27(18):5031-, 1992.

[2] Katia Genovese and Carmine Pappalettere. Whole 3D shape reconstruction of vascular segments under pressure via fringe projection techniques. Optics and Lasers in Engineering, 44(12):1311-1323, December 2006.

[3] R. I. Hartley and A. Zisserman. Multiple View Geometry in Computer Vision. Cambridge University Press, ISBN: 0521540518, second edition, 2004.

[4] M Arthington, C Siviour, B Elliott and N Petrinic. Cross section reconstruction during uniaxial loading, submitted to Measurement Science and Technology February 2009

[5] Todd M Osman. ASM Handbook, volume 8, page 3:12. American Society for Metals, 12 edition, 2000 . 